\title{
Pericardiectomy for constrictive pericarditis with postoperative increase of tricuspid regurgitation
}

\author{
Shinichi Taguchi*, Osamu Ishida, Atsuo Mori and Ryo Suzuki \\ *Correspondence: staguchi@z3.keio.jp \\ Department of Cardiovascular Surgery, Kawasaki Municipal Hospital, Kawasaki-city, Japan.
}

\begin{abstract}
We report a case of tricuspid regurgitation (TR) that increased markedly after pericardiectomy for constrictive pericarditis. Preoperative mild-to-moderate TR increased to severe following surgery. The patient was asymptomatic, and gradual regression of TR was observed. Eighteen months postoperatively, a left atrial thrombus formed, and a second surgery consisting of thrombectomy and tricuspid annuloplasty was performed. The increase in TR after pericardiectomy was thought to be due to dilatation of the right chambers and the annulus of the tricuspid valve. Several studies that entail mitral regurgitation after pericardiectomy are discussed.
\end{abstract}

Keywords: Pericardiectomy, constrictive pericarditis, tricuspid regurgitation, mitral regurgitation

\section{Introduction}

Pericardiectomy for constrictive pericarditis is not a complicated procedure; it involves peeling off the hard shell of calcification. However, it is not always a safe procedure and has a $5-15 \%$ perioperative mortality [1]. Longstanding disease may induce myocardial atrophy or fibrosis; mediastinalin flammation and fibrosis may produce recurrent cardiac compression, and inadequate or incomplete resection may result in no improvement [1]. Tricuspid regurgitation (TR) is another factor, which may complicate pericardial constriction [2]. Recently, we encountered a surgical case of pericardial constriction in which TR increased postoperatively. Although the patient tolerated the early-phase procedure, tricuspid annuloplasty was subsequently performed. The relationship between pericardiectomy and regurgitation of the atrio-ventricular valves is discussed in this report.

\section{Case presentation}

A 68-year-old woman was referred to our hospital due to acute heart failure. She had been diagnosed with constrictive pericarditis with extensive calcification 15 months previously. After treating the acute heart failure with intravenous furosemide, her symptoms abated; however, she required a high dosage of oral furosemide $(120 \mathrm{mg} /$ day $)$ and spironolactone $(50 \mathrm{mg} /$ day). She was scheduled for surgery in June 2009. Preoperative transthoracic echocardiography revealed diastolic dysfunction with a mild to moderate TR. The left ventricular diastolic dimension (LVDd) was $40 \mathrm{~mm}$, the left ventricular systolic dimension (LVDs) was $27 \mathrm{~mm}$, and the left ventricular ejection fraction (LVEF) was $60.0 \%$. The deceleration time was $127 \mathrm{msec}$, indicating diastolic dysfunction. (Table 1) presentsthe results of echocardiograms in order from preoperative to recent dates.
Cardiac catheterization revealed a dip and plateau of right and left ventricular pressures. Computed tomography (CT) revealed severe calcification of the pericardium.

The first surgery was a pericardiectomy, via amedian sternotomy, and cardiopulmonary bypass(CPB). The anterior pericardium was incised between the phrenic nerves and the inferior pericardium. The harmonic scalpel was effective in resecting the calcified pericardial shell. Since immediate preoperative echocardiography revealed only mild TR, tricuspid repair was not performed. Postoperative echocardiography revealed severe TR due to annular dilatation. The LVDd increased to $47 \mathrm{~mm}$, the LVDs increased to $34 \mathrm{~mm}$, and the LVEF decreased to $54.9 \%$. Although she still required the same doses of oral diuretics, she had no cardiac failure symptoms, and received routine follow-up. (Figure 1) shows that TR increased after the first surgery. (Figure 2) shows the preoperative enhanced CT and the postoperative plain $\mathrm{CT}$. The annular diameter of the tricuspid valve increased from $33.8 \mathrm{~mm}$ to $36.9 \mathrm{~mm}$ postoperatively. After pericardiectomy, the direction of the apex rotated dorsally, and the right heart chambers moved anteriorly.

A pericardial biopsy revealed no signs of active inflammation and no signs of granuloma formation. Therefore, the etiology of the pericarditis was unknown. Furthermore, cultures were negative for bacterial infection and negative for tuberculous and nontuberculous mycobacterial infection.

The preoperative serum creatine kinase (CK)-MB of the referral day when acute cardiac failure occurred was only 18 IU/I. In addition, the serum CK-MB on the first postoperative day was only $26 \mathrm{IU} / \mathrm{I}$. These data indicated that with the first procedure there was no evidence of myocardial involvement pre- and post-surgery.

In December 2010, echocardiography revealed moderate TR 
Taguchi et al. Cardiovascular System 2013,

http://www.hoajonline.com/journals/pdf/2052-4358-1-9.pdf

doi: 10.7243/2052-4358-1-9

Table 1. Echocardiography.

\begin{tabular}{llllll}
\hline & Pre $^{\text {st }} \mathbf{~ o p ~}$ & Post $\mathbf{1}^{\text {st }}$ op & Pre $\mathbf{2}^{\text {nd }}$ op & Post $\mathbf{2}^{\text {nd }}$ op & Recent \\
\hline $\begin{array}{l}\text { LVDd } \\
(\mathbf{m m})\end{array}$ & 40 & 47 & 49 & 65 & 55 \\
$\begin{array}{l}\text { LVDs } \\
(\mathbf{m m})\end{array}$ & 27 & 34 & 31 & 52 & 37 \\
$\begin{array}{l}\text { LVEF } \\
(\%)\end{array}$ & 60.0 & 54.9 & 66.8 & 40.7 & 59.7 \\
TR & mild-moderate & severe & moderate & trivial & mild \\
MR & trivial & trivial & trivial & trivial & mild \\
\hline
\end{tabular}

LVDd: Left Ventricular Diastolic Dimension

LVDS: Left Ventricular Systolic Dimension

LFEF: Left Ventricular Ejection Fraction

TR: Tricuspid Regurgitation

MR: Mitral Regurgitation
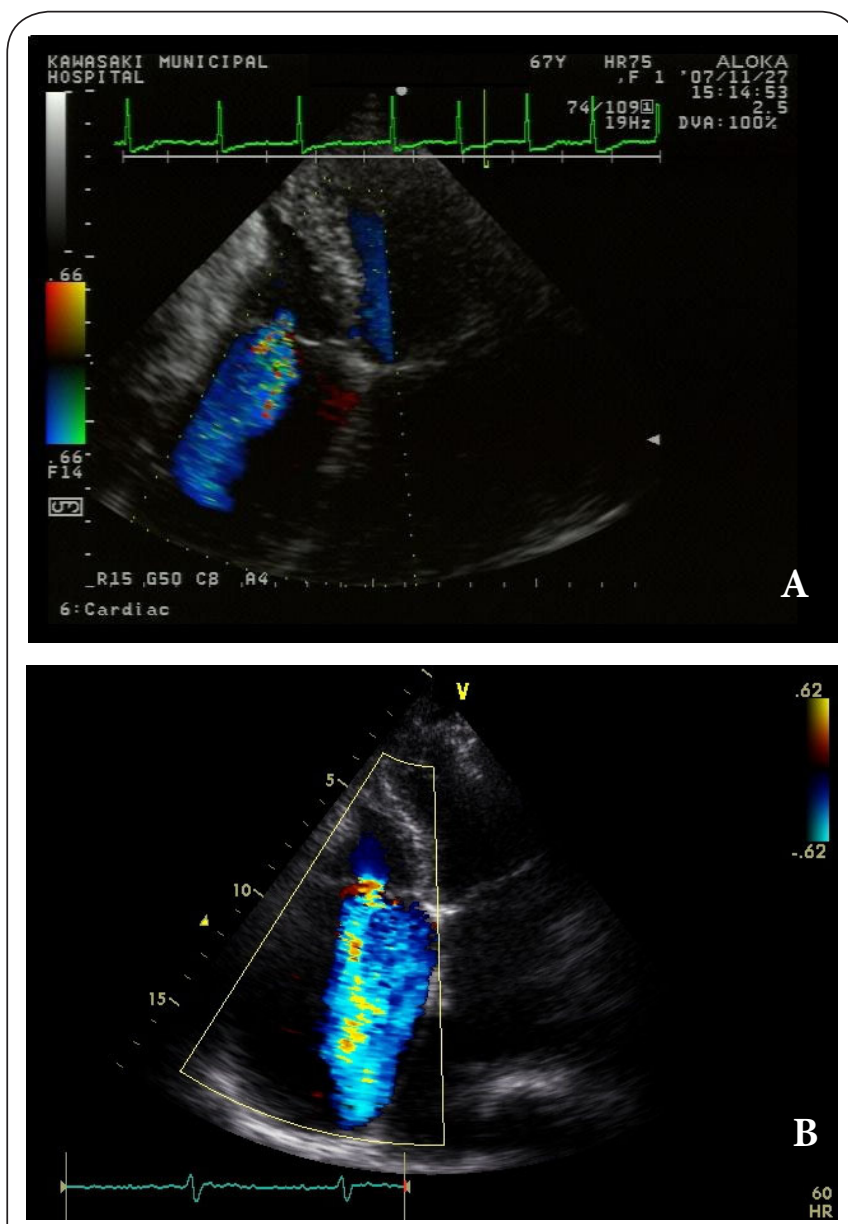

Figure 1. (A) Preoperative color Doppler echocardiography showing mild to moderate tricuspid regurgitation (TR).

(B) Postoperative color Doppler echocardiography showing severe TR.

with a $3 \mathrm{~cm}$ thrombus in the left atrium. The etiology of the thrombus formation was suspected to be the enlarged left atrium $(63 \mathrm{~mm})$, atrial fibrillation, and suboptimal warfarin
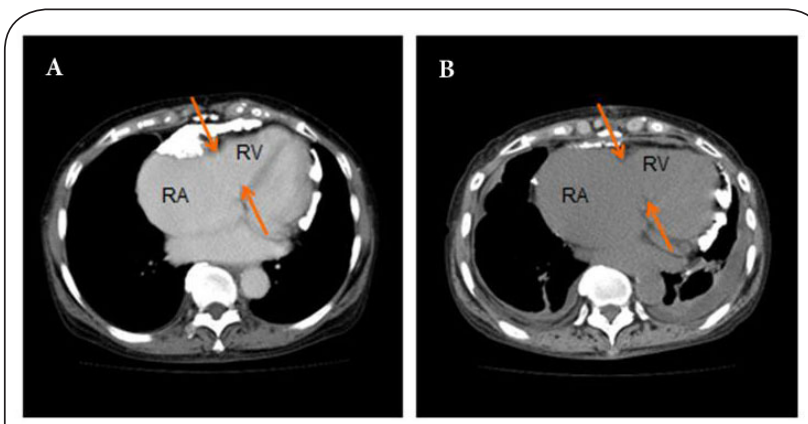

Figure 2. RA: right atrium; RV: right ventricle.

(A) Preoperative enhanced computed tomography (CT).

The arrows indicate the tricuspid annulus. The distance between the arrows measures $33.8 \mathrm{~mm}$.

(B) Postoperative plain CT. The arrows indicate the tricuspid annulus. The distance between the arrows measures $36.9 \mathrm{~mm}$. The annular diameter of the tricuspid valve increased postoperatively.

control (PT-INR 1.20). The LVDd was $49 \mathrm{~mm}$, the LVDs was $31 \mathrm{~mm}$, and the LVEF was $66.8 \%$. The second surgery consisted of a tricuspid annuloplasty using a $34 \mathrm{~mm}$ Cosgrove ring and a thrombectomy. Postoperatively, transient renal failure occurred, and echocardiography revealed trivial TR. The LVDd increased to $65 \mathrm{~mm}$, the LVDs increased to $52 \mathrm{~mm}$, and the LVEF decreased to $40.7 \%$.

The patient is currently doing well; echocardiography reveals mild TR, the LVDd is $55 \mathrm{~mm}$, the LVDs is $37 \mathrm{~mm}$, and the LVEF is $59.7 \%$. During the course of follow-up, mitral regurgitation (MR) has remained trivial, except for the recent echocardiography, which revealed mild MR.

\section{Discussion}

There are two standard approaches for a pericardiectomy: anantero-lateral thoracotomy or a median sternotomy [3]. We usually select a median sternotomy with routine use of CPB. We believe that it safer to use CPB because of the potential risks of hemodynamic deterioration with this disease. Under a median sternotomy, a nanterior excision of the pericardium between the phrenic nerves, and the inferior pericardium is performed. This primarily relieves the surface of the right chambers and some of the left chambers, which may lead to annular dilatation and regurgitation of the tricuspid valve.

Gongora et al., [2] evaluated 261 cases of constrictive pericarditis. Among those with moderate/severe TR, operative mortality was similar whether or not repair was undertaken. They reported only $29 \%$ improvement in TR if repair was not performed. Their conclusion was to consider repair when moderate/severe TR exists to reduce symptoms. TR may worsen after pericardiectomy [2,4]. Our case exhibited only mild TR immediately before surgery, and mild cases were not discussed by Gongora et al.

Mantri et al., [5] studied 14 cases of pericardiectomy for 
constrictive pericarditis with TR or MR without repairing them. Long term follow-up resulted in regression of the regurgitation in half the cases. They concluded that if an adequate pericardiectomy is done at an earlier stage, the regurg-itations will regress. There were no patients in whom these regurgitations worsened after pericardiectomy.

Johnson et al., [4] described two cases of pericardiectomy in which TR worsened postoperatively. They suggested that the deterioration was a result of postoperative right ventricular dilatation. Expansion of the right ventricle results in severe TR. In our case as well, expansion of the right ventricle due to decortification anteriorly and the annular dilatation of the tricuspid valve occurred concomitantly.

We did not perform tricuspid annuloplasty because the echocardiography taken immediately before the first surgery showed only mild TR. Since severe TR developed postoperatively, tricuspid annuloplasty was probably indicated in the first surgery. Before the second surgery, TR had decreased to a moderate level. Since the second surgery was indicated due to a left atrial thrombus, concomitant tricuspid annuloplasty was performed. There are cases in which MR after pericardiectomy subsided due to compensation and did not require reoperation $[6,7]$. Similarly in our case, the possibility exists that the TR might have regressed further without the second surgery.

Nakamura et al., [8] reported a case of severe MR postoperatively that required surgical management three weeks after the pericardiectomy. Their case was primary mitral disease; thus, it differs from that of ours. They stated that in MR after pericardiectomy, the change in the left ventricular geometry and shift of the papillary muscles [6], increased mobility of the lateral wall, and increased the left ventricular volume [7], are the hypotheses for the cause. Although our case was a TR, the same hypotheses may be applied to the right ventricle.

After pericardiectomy, initial clinical and hemodynamic responses are not always dramatic and continued improvement may occur over many months [9]. This is consistent with the fact that the left ventricular dimension progressively increases and the systolic function remains stable [9]. Since the left ventricle is located in the postero-lateral side where the thick pericardium is not resected, it is likely that the left ventricle gradually dilates. In our case, the most recent left ventricular dimension was larger than the preoperative data from the first procedure. This may also explain why the MR recently increased from trivial to mild with probable annular dilatation.

\section{Conclusions}

TR increased markedly after pericardiectomy for constrictive pericarditis. The etiology is thought to be the dilatation of the right chambers with annular dilatation of the tricuspid valve. In treating constrictive pericarditis, care must be taken to evaluate TR, even if it is mild. Even if TR deteriorates to a severe level, it may regress due to compensation.

\section{Competing interests}

The authors declare that they have no competing interests.

\section{Authors' contributions}

ST and AM contributed to the preoperative planning and the surgical procedures. Ol was involved in data collection. All authors contributed to case follow-up. All authors read and approved the final manuscript.

\section{Publication history}

Editor: Thomas L. Higgins, Tufts University School of Medicine, USA. EIC: William Clifford Roberts, Baylor University Medical Center, USA. Received: 10-May-2013 Revised: 10-Aug-2013

Accepted: 21-Aug-2013 Published: 29-Aug-2013

\section{References}

1. LeWinter MM and Tischler MD. Pericardial diseases. In Bonow RO, Mann DL, Zipes DP and Libby P(Eds), Braunwald's Heart Disease 2012; 1651-71. I Book

2. Gongora E, Dearani JA, Orszulak TA, Schaff HV, Li Z and Sundt TM. Tricuspid regurgitation in patients undergoing pericardiectomy for constrictive pericarditis. Ann Thorac Surg. 2008; 85:163-70. | Article | PubMed

3. Maisch B, Seferovic PM, Ristic AD, Erbel R, Rienmuller R, Adler Y, Tomkowski WZ, Thiene $\mathrm{G}$ and Yacoub $\mathrm{MH}$. Guidelines on the diagnosis and management of pericardial diseases executive summary; The Task force on the diagnosis and management of pericardial diseases of the European society of cardiology. Eur Heart J. 2004; 25:587-610. | Article I PubMed

4. Johnson TL, Bauman WB and Josephson RA. Worsening tricuspid regurgitation following pericardiectomy for constrictive pericarditis. Chest. 1993; 104:79-81. | Article I PubMed

5. Mantri RR, Radhakrishnan S, Sinha N, Goel PK, Bajaj R and Bidwai PS. Atrio-ventricular regurgitations in constrictive pericarditis: incidence and post-operative outcome. Int J Cardiol. 1993; 38:273-9. | Article I PubMed

6. Buckingham RE, Jr., Furnary AP, Weaver MT, Floten HS and Davis RF. Mitral insufficiency after pericardiectomy for constrictive pericarditis. Ann Thorac Surg. 1994; 58:1171-4. I Article I PubMed

7. Terada Y, Mitsui T and Yamada S. Mitral regurgitation after pericardiectomy for constrictive pericarditis. Jpn J Thorac CardiovasC Surg. 1999; 47:27-30. | Article I PubMed

8. Nakamura T, Masai T, Yamauchi T, Higuchi T, Ito H, Toyoshima $Y$ and Sawa Y. Successful surgical management for severe mitral regurgitation unmasked after pericardiectomy for chronic constrictive pericarditis. Ann Thorac Surg. 2008; 86:1994-6. | Article | PubMed

9. Senni M, Redfield MM, Ling LH, Danielson GK, Tajik AJ and Oh JK. Left ventricular systolic and diastolic function after pericardiectomy in patients with constrictive pericarditis: Doppler echocardiographic findings and correlation with clinical status. J Am Coll Cardiol. 1999; 33:1182-8. | Article | PubMed

\section{Citation:}

Taguchi S, Ishida O, Mori A and Suzuki R. Pericardiectomy for constrictive pericarditis with postoperative increase of tricuspid regurgitation. Cardio Vasc Syst. 2013; 1:9

http://dx.doi.org/10.7243/2052-4358-1-9 